Check for updates

Cite this: Mater. Adv., 2022, 3,3286

Received 19th November 2021 Accepted 14th February 2022

DOI: $10.1039 / \mathrm{d} 1 \mathrm{ma} 01095 f$

rsc.li/materials-advances

\title{
Aggregation induced delayed green fluorescence from assembly of gold nanoclusters: an advanced probe for "background free" pyrophosphate recognition $\dagger$
}

\author{
Santanu Dolai, Srestha Basu* and Anumita Paul (D)*
}

\begin{abstract}
Aggregation induced emission (AIE), ever since its inception, has received remarkable attention from scientists pursuing research towards the development of advanced optical materials. Thus, a plethora of light emitting materials have been reported, based on the principle of AIE, as functional components in light emitting diodes and cell imaging probes. On the other hand, recent advancement in the field of organic electronics has been witnessed through rational implementation of the concept of delayed fluorescence (DF). Due to their long luminescence lifetime, DF materials also portend to be ideal candidates for elimination of the interference of auto fluorescence and thereby allow accurate sensing of chemical species. Thus, development of AIE-based DF materials may be a way forward in the field of molecular and ion recognition. Herein, we report that non-luminescent gold nanoclusters, upon complexation reaction with zinc ions, exhibit delayed fluorescence with a quantum yield as high as $6 \%$ and an average lifetime of $17.67 \mu$ s. The delayed green fluorescence of the aggregates of gold clusters was further used for "background free" probing of pyrophosphate ions.
\end{abstract}

\section{A Introduction}

The emergence of the concept of delayed fluorescence (DF) has been proposed to have revolutionized the science and technology pertinent to light emitting diodes (LEDs). ${ }^{1-11}$ It is worth mentioning here that delayed fluorescence materials have the potential to facilitate reverse intersystem crossing of photoexcited electrons from the triplet excited state (lowest) to the singlet state, wherein the gap between the singlet and triplet energy states is thermally accessible. ${ }^{12}$ This not only augments the luminescence quantum yield of an emitter but also enhances its excited state luminescence lifetime. Thus, in addition to fabrication of LEDs, DF emitters have been popular choices as lifetime imaging probes. ${ }^{13-18}$ Nevertheless, it may be noted here that TADF is not essentially related to the luminescence quantum yield. However, DF emitters in aggregated states often show a low quantum yield - as a consequence of which substantial chemical modification of the system is required. ${ }^{19}$ A promising entrant in the family of DF based materials is the one that exhibits aggregation induced delayed fluorescence (AIDF) ${ }^{19-21}$ Thus, designing AIDF based materials may not only overcome the limitations

Department of Chemistry, Indian Institute of Technology Guwahati, Guwahati, Assam - 781039, India.E-mail: anumita@iitg.ac.in, srestha@iitg.ac.in $\dagger$ Electronic supplementary information (ESI) available. See DOI: 10.1039/ d1ma01095f featured by conventional DF materials but may also offer a new genre of chemical species with tunable optical properties depending on the extent of aggregation.

Long persistent luminescence-based imaging probes are devoid of interference from short lived background auto fluorescence and offer an improved signal to noise ratio as opposed to conventional luminophores associated with scattering interference. On the other hand, an important domain where DF materials, especially AIDF molecules, may find applications is that with regard to sensing of important molecules. Traditional luminescence-based sensors have a "short" response time and hence suffer from the interference of background scattering and auto fluorescence. This limits the sensitivity and specificity of fluorescence-based detection and discrimination of analytes. In an allied vein, phosphorescent materials with adequate luminescence lifetime capable of overcoming the aforementioned limitations are typically constituted of heavy metal atoms and are hence not desirable for bio sensing of molecules. Furthermore, use of such heavy metal atom-based materials may lead to environmental concerns. This necessitates the development of DF materials with sufficient luminescence lifetime and quantum yield to emerge as futuristic chemosensors.

In this regard, a few important literature reports are available in which DF based sensing of hydrogen peroxide and the microenvironment has been pursued. ${ }^{22}$ In an allied vein, 
delayed fluorescence and prompt fluorescence have been simultaneously used at a single molecular level to understand the polarity of chemical systems. ${ }^{23}$ Furthermore, generalized protocols have been established for loading of TADF emitters onto polystyrene based nanoparticles for live cell imaging. ${ }^{24}$ Moreover, high molecular weight polymers with low dispersity were prepared using TADF emitting monomers and an oxadiazole acceptor core via a $\mathrm{Cu}(0)$ reversible deactivation radical polymerization process. These materials were further used for ratiometric sensing of oxygen. ${ }^{25}$ These studies suggest the need for further research in this field and hence, the emergence of DF based chemosensors with a long luminescence lifetime is deemed important. In addition, the aforementioned chemosensors are primarily organic in nature and hence are likely to suffer from limitations of poor photo-stability and biocompatibility. To this end, the rational design of nanoscale particle-based DF chemosensors with prolonged luminescence lifetime may be deemed to be the best of all options. Notably, aggregation of nanoclusters has been demonstrated to be an effective strategy to tune their optical properties. Recently, metal ion-induced aggregation of atomic clusters has been reported to be widely used for sensing (and imaging) of a plethora of analytes. $^{26-34}$ Thus, harnessing the delayed fluorescence properties of atomic clusters via chemical reaction-based aggregation may emerge to be an interesting avenue towards fabrication of newer optical materials.

To this end, oxoanions of phosphates are known to play critical roles in several biological processes ranging from signal processing and membrane transport to energy transduction and storage of genetic information. Notably, pyrophosphate ions, the product of hydrolysis of adenosine triphosphate (ATP), are also important disease markers. For example, in diseases like urolithiasis and osteoarthritis, the level of pyrophosphate is known to get elevated, thereby indicating health dysfunction. Furthermore, gauging intracellular pyrophosphate levels has also been recognized as a tool for cancer diagnostics. ${ }^{35}$ Thus "background free" recognition of pyrophosphate ions using robust chemical probes is deemed essential.

Herein we report unprecedented delayed fluorescence based selective sensing of pyrophosphate by metal ion mediated aggregates of gold clusters. Zinc ion assisted aggregates of gold nanoclusters (Zn Au NCs) were found to exhibit delayed fluorescence with a quantum yield of $6 \%$ and an average lifetime of $17.65 \mu \mathrm{s}$. Interestingly upon interaction with pyrophosphate ions the delayed fluorescence of $\mathrm{Zn} \mathrm{Au} \mathrm{NCs} \mathrm{was} \mathrm{found} \mathrm{to}$ quench completely when the concentration of pyrophosphate exceeded $2910 \mu \mathrm{M}$. Moreover, the chemical interaction between Zn Au NCs and pyrophosphate was found to be highly selective as complex anions such as thiosulphate, nitrate, and sulphate led to a slight change in the delayed luminescence of $\mathrm{Zn} \mathrm{Au} \mathrm{NCs.} \mathrm{The} \mathrm{transmission} \mathrm{electron} \mathrm{microscopy} \mathrm{analysis}$ (TEM) revealed that the delayed luminescence quenching upon the addition of pyrophosphate occurred due to disintegration of the zinc mediated aggregates of gold clusters. Thus the study embodied herein provides a unique avenue for "background free" detection of pyrophosphate, which is an important biological challenge worthy of pursuit.

\section{B Results and discussion}

Addition of mercaptopropanoic acid to a solution of tetrachloroauric acid led to the formation of a colourless dispersion. The UV-vis spectrum of this colourless dispersion was found to be featureless negating the formation of gold nanoparticles (Fig. S1A, ESI $\dagger$ ). Instead, TEM analysis of this colourless dispersion showed the presence of ultra-small particle sizes of $\sim 2 \mathrm{~nm}$ (Fig. S1B, ESI $\dagger$ ). The colourless dispersion, however, did not exhibit discernible luminescence upon excitation at $300 \mathrm{~nm}$ (Fig. S1C, ESI $\dagger$ ). Interestingly, upon the addition of $200 \mathrm{mg}$ of zinc acetate dihydrate to $10 \mathrm{~mL}$ of this solution, a yellow coloured dispersion was formed which exhibited broad absorbance over the entire UV-visible range (Fig. 1C and F1) and was highly luminescent under a $365 \mathrm{~nm}$ lamp (Fig. 1F2). The delayed luminescence spectrum of this dispersion acquired upon eliminating all the luminescence events in the nanosecond range featured a strong emission peak centered at $495 \mathrm{~nm}$ upon excitation at $300 \mathrm{~nm}$ (Fig. 1A and B). The average lifetime of the delayed fluorescence at $495 \mathrm{~nm}$ from $\mathrm{Zn}$ Au NCs was determined to be $17.65 \mu$ s (Fig. 1D and Table S1, ESI $\dagger$ ). TEM analysis indicated that the dispersion comprised of aggregates of gold clusters, possibly mediated by zinc ions. In order to confirm the co-presence of the gold and zinc elements on the aggregates, elemental mapping was performed (Fig. S2A-D, $\mathrm{ESI} \dagger$ ). It is noteworthy that in addition to the delayed spectrum, regular fluorescence from $\mathrm{Zn} \mathrm{Au}$ NCs with an emission at $495 \mathrm{~nm}$ (Fig. S3, ESI $\dagger$ ) was also observed with an average decay lifetime of 10.35 ns (Table S2, ESI $\dagger$ ). The average size of the $\mathrm{Zn}$ $\mathrm{Au}$ NCs was calculated to be $26 \pm 0.28 \mathrm{~nm}$. Thus, owing to the rather uniform size of the aggregates of $\mathrm{Au}$ NCs, the possible effect of the size dependent alteration in luminescence features of $\mathrm{Zn} \mathrm{Au} \mathrm{NCs} \mathrm{may} \mathrm{be} \mathrm{negligible} \mathrm{(Fig.} \mathrm{S4,} \mathrm{ESI} \dagger$ ).

Moreover, in order to substantiate the phenomenon of DF from the metal ion mediated aggregation of clusters, further photo-physical experiments were pursued. Specifically, to confirm the involvement of triplet states in the emission from $\mathrm{Zn}$

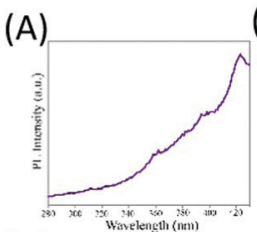

(D)

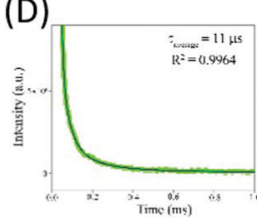

(B)

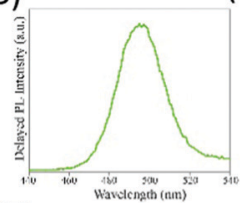

(E)

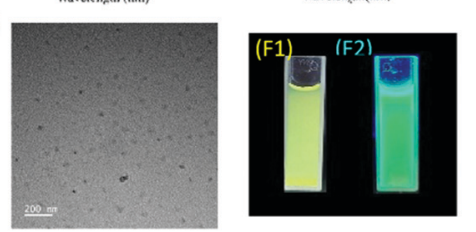

(C)

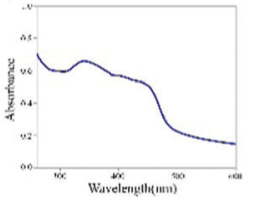

Fig. 1 (A) Delayed excitation spectrum of Zn Au NCs upon fixing the emission at $494 \mathrm{~nm}$. (B) Delayed emission spectrum of Zn Au NCs upon fixing the excitation at $300 \mathrm{~nm}$. (C) UV-vis absorbance spectrum of $\mathrm{Zn} \mathrm{Au}$ NCs. (D) Time resolved delayed photoluminescence decay curve of $\mathrm{Zn} \mathrm{Au}$ NCs. (E) Transmission electron microscopy image of Zn Au NCs. Digital photographs of Zn Au NCs under (F1) broad daylight and (F2) UV excitation of $365 \mathrm{~nm}$ 

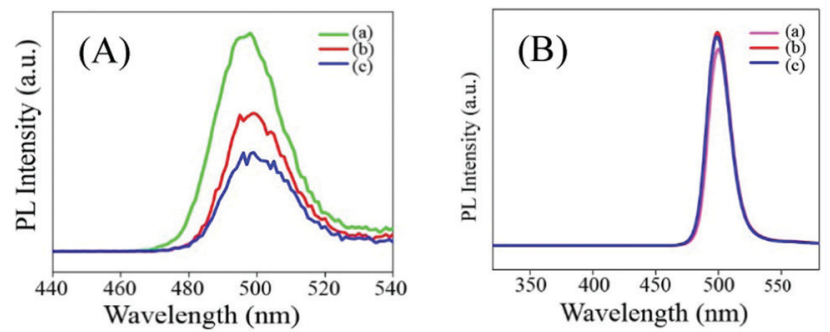

(C)

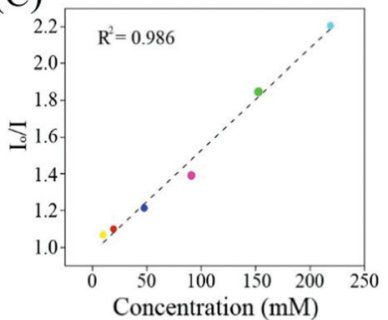

Fig. 2 (A) Delayed fluorescence emission spectrum of (a) Zn Au NCs and that following the addition of (b) $220 \mu \mathrm{L}$ and (c) $560 \mu \mathrm{L}$ addition of $1 \mathrm{M} \mathrm{KI}$ solution. (B) Prompt fluorescence emission spectrum of (a) Zn Au NCs and that following the addition of (b) $220 \mu \mathrm{L}$ and (c) $560 \mu \mathrm{L}$ addition of $1 \mathrm{M} \mathrm{KI}$ solution. (C) Stern Volmer plot calculated based on (A).

$\mathrm{Au}$ NCs, a typical triplet state quencher i.e. iodide ions were added to an aqueous dispersion of $\mathrm{Zn} \mathrm{Au} \mathrm{NCs.} \mathrm{This} \mathrm{led} \mathrm{to}$ significant quenching of the $\mathrm{DF}$ of $\mathrm{Zn} \mathrm{Au} \mathrm{NCs} \mathrm{(Fig.} \mathrm{2A).}$
However, negligible quenching was observed in prompt fluorescence of $\mathrm{Zn}$ Au NCs upon the addition of iodide ions (Fig. 2B). The Stern Volmer quenching constant of the DF of Zn Au NCs upon the addition of KI was calculated to be $3.157 \times 10^{5}$. This clearly highlighted the involvement of triplet states in the emission from $\mathrm{Zn} \mathrm{Au} \mathrm{NCs.} \mathrm{Furthermore,} \mathrm{the} \mathrm{possibility} \mathrm{of}$ phosphorescence may possibly be ruled out as no discernible bathochromic shift was observed in the delayed fluorescence of $\mathrm{Zn}$ Au NCs in comparison to prompt fluorescence.

Emission in nanoclusters has been proposed to originate from ligand to metal charge transfer. This charge transfer process occurs in a certain time range which corresponds to the luminescence lifetime of the emission process. However, when the clusters are assembled into nanostructures comprising many such cluster units the charge transfer is likely to occur through a series of clusters and ligands present in the assembled structure. Thus as opposed to non-assembled clusters, the charge transfer process in assembled clusters is likely to occur over a much longer time scale, thereby accounting for the increase in the emission lifetime. In the current system of $\mathrm{Au}$ NCs assembled by $\mathrm{Zn}$ ions, this maybe the origin of delayed luminescence.

Another possibility may be the facile deactivation of the excited states of non-assembled clusters due to the relaxation processes associated with the ligands stabilizing them. However, upon formation of rigid assemblies such relaxation

(A)

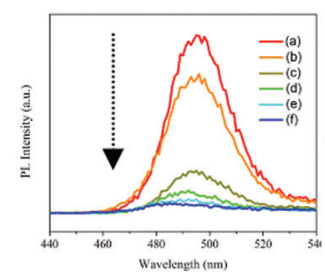

\section{(B)}

(C)

(D)

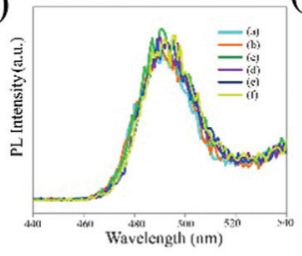

(E)

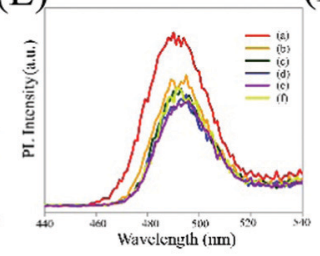

(G)

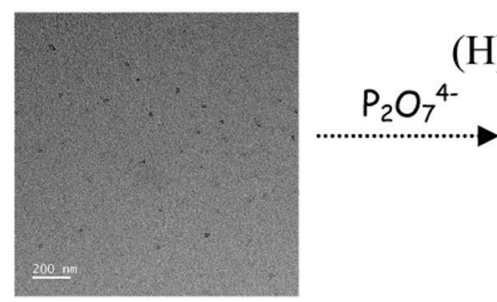

(F)
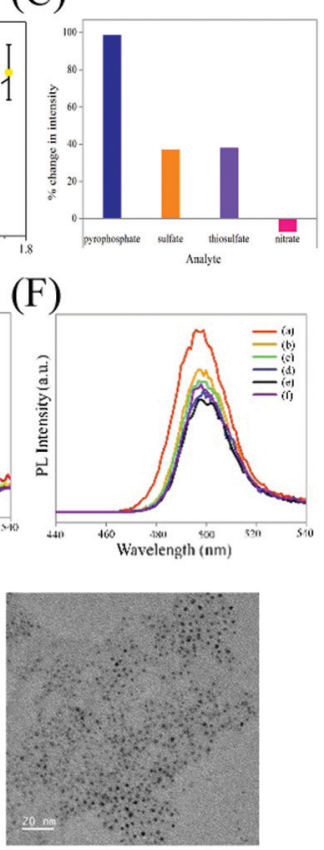

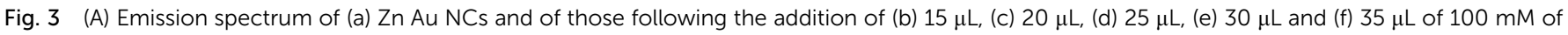

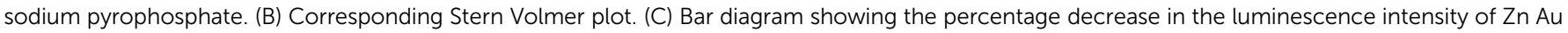

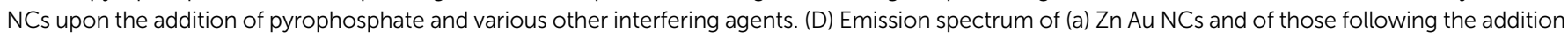

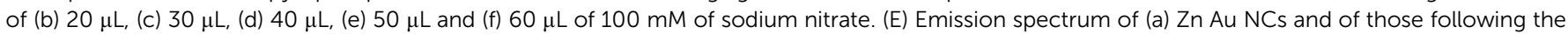

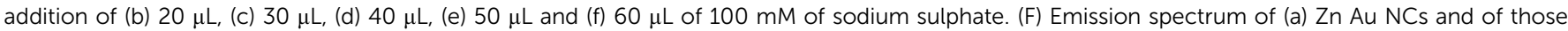

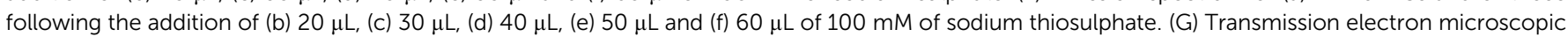
image of $\mathrm{Zn}$ Au NCs and $(\mathrm{H})$ of that following the addition of $30 \mu \mathrm{L}$ of $100 \mathrm{mM}$ sodium pyrophosphate. 
processes are highly restricted thereby prolonging the life time of the excited states of the clusters and making them available for ISC and reverse ISC. ${ }^{36}$

In the next step, the delayed luminescence from $\mathrm{Zn}$ Au NCs was used as a probe to monitor the chemical interaction between pyrophosphate and zinc ion assembled gold nanoclusters. Intriguingly, upon the addition of pyrophosphate ions to a dispersion of $\mathrm{Zn} \mathrm{Au} \mathrm{NCs} \mathrm{the} \mathrm{delayed} \mathrm{fluorescence} \mathrm{at} 495 \mathrm{~nm}$ was found to quench (Fig. 3A) with a concomitant blue shift of $\sim 15 \mathrm{~nm}$. The corresponding decrease in the relative intensity of the delayed luminescence of $\mathrm{Zn} \mathrm{Au} \mathrm{NCs} \mathrm{with} \mathrm{the} \mathrm{added} \mathrm{concentration} \mathrm{of}$ pyrophosphate ions was found to obey the Stern-Volmer relationship (Fig. 3B) with a Stern-Volmer constant of $10.728 \times 10^{-3} \mathrm{M}^{-1}$. This value of Stern-Volmer constant along with the $17.67 \mu \mathrm{s}$ average lifetime for delayed luminescence of $\mathrm{Zn}$ Au NCs yielded a quenching constant of $6.07 \times 10^{8} \mathrm{M}^{-1} \mathrm{~s}^{-1}$ of the delayed luminescence of $\mathrm{Zn} \mathrm{Au} \mathrm{NCs} \mathrm{with} \mathrm{pyrophosphate.} \mathrm{On} \mathrm{the} \mathrm{other}$ hand, chemical interaction (if any) with other poly anions, such as nitrate, sulphate and thiosulphate led to a much subdued effect on the delayed luminescence of $\mathrm{Zn}$ Au NCs (Fig. 3C-F).

In order to gain insight into the mechanism driving the change in delayed luminescence in $\mathrm{Zn} \mathrm{Au}$ NCs upon the addition of pyrophosphate, TEM analysis was pursued. Interestingly, it was observed that zinc mediated aggregated gold clusters underwent significant disaggregation upon the addition of pyrophosphate ions (Fig. 3G and H). This may be attributed to the fact that the zinc ions, assembling the gold clusters, upon the addition of pyrophosphate, underwent chemical interaction with the latter. This might have led to the disaggregation of the gold clusters causing a sharp decrease in the delayed luminescence which was otherwise observed only in the presence of zinc ions. Evidence of this chemical interaction was observed in the changes in the FTIR spectrum of $\mathrm{Zn} \mathrm{Au} \mathrm{NCs} \mathrm{upon} \mathrm{the} \mathrm{addition} \mathrm{of} \mathrm{pyrophosphate} \mathrm{ions} \mathrm{(Fig.} \mathrm{S5}$ and Table S3, ESI $\dagger$ ). The FTIR spectra of $\mathrm{Zn} \mathrm{Au} \mathrm{NCs} \mathrm{show} \mathrm{two}$ strong and sharp features at 1390 and $1542 \mathrm{~cm}^{-1}$ due to the symmetric and asymmetric stretching modes of the carboxylate groups of MPA, which interact with $\mathrm{Zn}^{2+}$ resulting in aggregation of the gold nanoparticles. Upon the addition of pyrophosphate ions, these two peaks display features on their higher frequency edge. This blue shift is probably caused due to weakening interactions between the zinc ions with the carboxylate groups of MPA as a result of the stronger interaction of zinc cations with the added pyrophosphate anion. Furthermore, the selectivity of $\mathrm{Zn} \mathrm{Au} \mathrm{NCs} \mathrm{towards} \mathrm{other} \mathrm{phosphate-}$ containing compounds has been confirmed. For this, tetrabutylammonium hexafluorophosphate and disodium hydrogen phosphate were added to an aqueous dispersion of $\mathrm{Zn} \mathrm{Au}$ NCs, with a concentration akin to that of pyrophosphate. Intriguingly, while for a particular concentration of pyrophosphate, the delayed luminescence intensity of Zn Au NCs was quenched completely, the effect of tetrabutylammonium hexafluorophosphate and disodium hydrogen phosphate on the delayed luminescence intensity of $\mathrm{Zn} \mathrm{Au}$ NCs remained much less pronounced (Fig. S6, ESI $\dagger$ ). Furthermore, selective sensing of pyrophosphate was achieved in the presence of tetrabutylammonium hexafluorophosphate and disodium hydrogen phosphate. For this, a mixture of tetrabutylammonium hexafluorophosphate and disodium hydrogen phosphate was added to an aqueous dispersion of $\mathrm{Zn} \mathrm{Au} \mathrm{NCs.} \mathrm{Interest-}$ ingly, a slight change in the delayed luminescence intensity of $\mathrm{Zn} \mathrm{Au} \mathrm{NCs} \mathrm{was} \mathrm{observed} \mathrm{upon} \mathrm{the} \mathrm{addition} \mathrm{of} \mathrm{the} \mathrm{same.}$ Thereafter, pyrophosphate ions were introduced into the dispersion of $\mathrm{Zn} \mathrm{Au} \mathrm{NCs} \mathrm{added} \mathrm{to} \mathrm{a} \mathrm{mixture} \mathrm{of} \mathrm{tetrabutylammo-}$ nium hexafluorophosphate and disodium hydrogen phosphate. Upon the addition of pyrophosphate, a significant decrease in luminescence intensity of $\mathrm{Zn} \mathrm{Au}$ NCs was observed (Fig. S7, ESI $\dagger$ ).

Now here it may be noted that although such visual probing of pyrophosphate ions by $\mathrm{Zn}$ Au NCs could be carried out by regular luminescence studies (without the $1 \mu$ s delay) (Fig. S8, ESI $\dagger$ ) these would be inherently associated with interference from background scattering. The Stern Volmer constant for normal fluorescence quenching by pyrophosphate ions was found to be $1.5102 \times 10^{+3} \mathrm{M}^{-1}$. Also, with an average lifetime of $10.35 \mathrm{~ns}$ the quenching constant is determined to be $1.459 \times 10^{11} \mathrm{M}^{-1} \mathrm{~s}^{-1}$. Thus, the study shown herein demonstrates the advantage of using delayed luminescence vis-à-vis normal luminescence in monitoring selectively the concentration of pyrophosphate ions in solution. In a nutshell, owing to strong interaction between zinc ions and pyrophosphate, upon the addition of pyrophosphate ions, $\mathrm{Zn} \mathrm{Au} \mathrm{NCs} \mathrm{undergo}$ disaggregation, leading to quenching of the delayed luminescence intensity of the latter (Scheme 1).

Furthermore, we have demonstrated that the concentration of pyrophosphate in human blood serum could be estimated using a $\mathrm{Zn} \mathrm{Au} \mathrm{NCs} \mathrm{probe.} \mathrm{For} \mathrm{this,} 50 \mu \mathrm{L}$ of serum was added to $2 \mathrm{~mL}$ of $\mathrm{Zn}$ Au NCs. The delayed fluorescence spectrum of $\mathrm{Zn}$ $\mathrm{Au}$ NCs was recorded in the presence of blood serum $\left(I_{0}\right)$. To this dispersion of $\mathrm{Zn} \mathrm{Au} \mathrm{NCs,} 40 \mu \mathrm{L}$ of pyrophosphate solution (70 $\mathrm{mM}$ ) was added. This led to quenching of the delayed luminescence of $\mathrm{Zn}$ Au NCs. Likewise, pyrophosphate ions of various concentrations such as $80,100,110$, and $120 \mathrm{mM}$, were added to $\mathrm{Zn} \mathrm{Au} \mathrm{NCs} \mathrm{(in} \mathrm{the} \mathrm{presence} \mathrm{of} \mathrm{blood} \mathrm{serum)} \mathrm{and}$ the extent of decrease in the delayed luminescence intensity $\left(I_{n}, n=70,80,100,110\right.$ and 120) of the Zn Au NCs was noted. Thereafter, $I_{n} / I_{0}$ was plotted against the corresponding concentration of pyrophosphate ions. This gave a linear curve which could be used as a calibration curve for the detection of an unknown amount of pyrophosphate ions in human blood

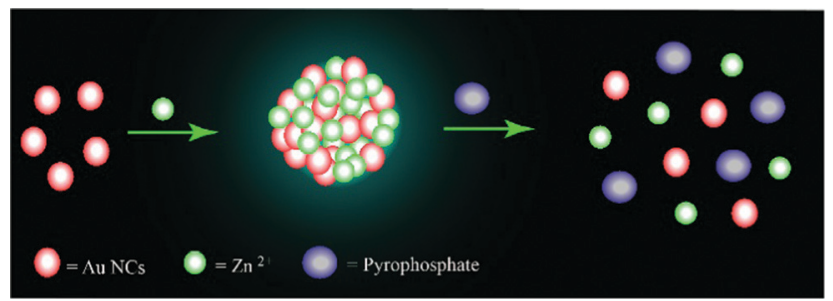

Scheme 1 Schematic representation of the possible mechanism of quenching of the delayed bright green luminescence of Zn Au NCs upon the addition of sodium pyrophosphate. 

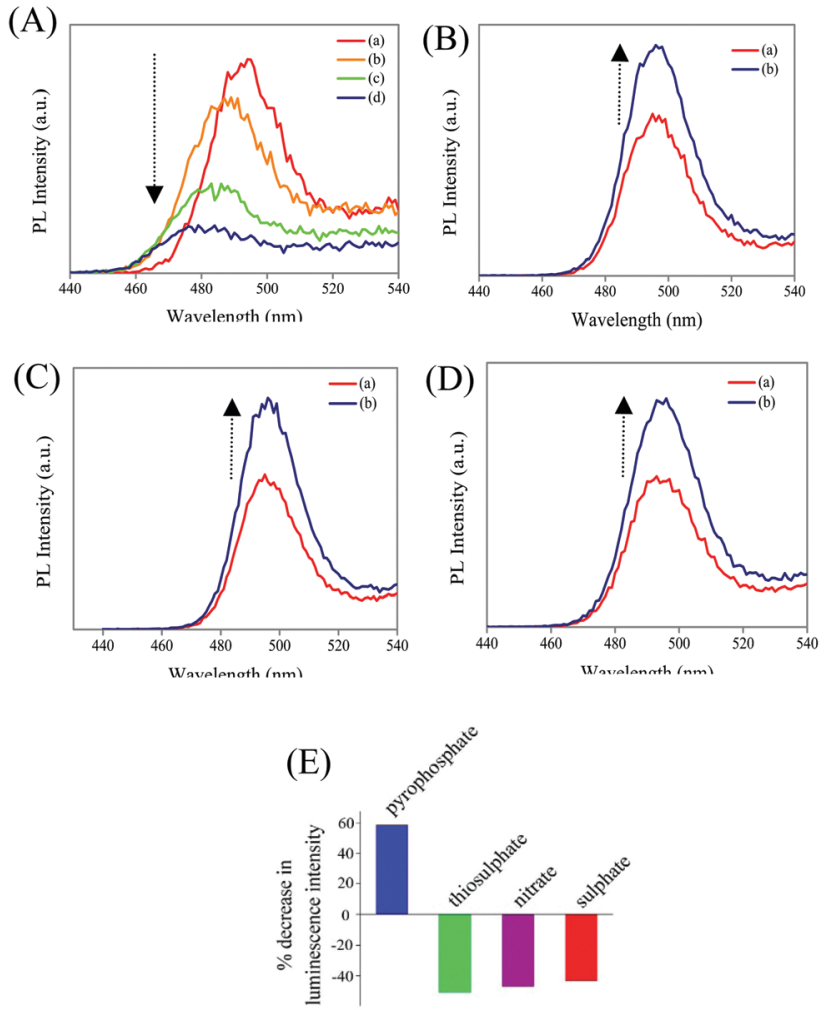

Fig. 4 (A) Emission spectrum of (a) Zn Au NCs in human blood serum and those following the addition of (b) $22.2 \mu \mathrm{L}$, (c) $32.2 \mu \mathrm{L}$ and (d) $42.2 \mu \mathrm{L}$ of pyrophosphate ions in human blood serum. (B) Emission spectrum of (a) Zn Au NCs in human blood serum and that following the addition of (b) $32.2 \mu \mathrm{L}$ of sulphate ions in human blood serum. (C) Emission spectrum of (a) Zn Au NCs in human blood serum and that following the addition of (b) $32.2 \mu \mathrm{L}$ of nitrate ions in human blood serum. (D) Emission spectrum of (a) $\mathrm{Zn} \mathrm{Au} \mathrm{NCs} \mathrm{in} \mathrm{human} \mathrm{blood} \mathrm{serum} \mathrm{and} \mathrm{that} \mathrm{following} \mathrm{the} \mathrm{addition} \mathrm{of}$ (b) $32.2 \mu \mathrm{L}$ of thiosulphate ions in human blood serum. (E) Bar diagram showing the percentage decrease in luminescence intensity of Zn Au NCs upon the addition of pyrophosphate and various other interfering agents in human blood serum.

serum. In order to demonstrate this, $40 \mu \mathrm{L}$ of pyrophosphate ions of unknown concentration was added to $\mathrm{Zn} \mathrm{Au} \mathrm{NCs} \mathrm{in}$ blood serum and the corresponding extent of decrease in the delayed luminescence intensity of $\mathrm{Zn}$ Au NCs was fitted into the calibration curve. The unknown concentration of pyrophosphate ions (in human blood serum) was determined to be $89 \mathrm{mM}$. The results are shown in Fig. S9 (ESI $\dagger$ ).

Furthermore, in order to elucidate the practical application of the designed sensor in biological media, delayed fluorescence-based detection of pyrophosphate ions was pursued in human blood serum. Interestingly, the presence of pyrophosphate ions could be detected in human blood serum with high selectivity and sensitivity (Fig. 4A). Importantly, common interfering ions like thiosulphate, sulphate and nitrate ions had discernibly different effects on the luminescence of $\mathrm{Zn} \mathrm{Au} \mathrm{NCs} \mathrm{and} \mathrm{were} \mathrm{found} \mathrm{not} \mathrm{to} \mathrm{intervene} \mathrm{in} \mathrm{the}$ detection of pyrophosphate ions (Fig. 4B-E). Furthermore, we have measured the delayed emission of $\mathrm{Zn}$ Au NCs upon the addition of pyrophosphate ions in a mixture of thiosulphate, sulphate and nitrate anions. Interestingly, with the increasing concentration of pyrophosphate anions, the delayed emission of $\mathrm{Zn} \mathrm{Au}$ NCs was found to decrease in a manner akin to the absence of the above-mentioned interfering ions (Fig. S10, ESI $\dagger$ ).

\section{Materials and methods}

\section{Synthesis of gold nanoclusters}

Gold nanoclusters were synthesized following the addition of $0.4 \mathrm{~mL}$ of mercaptopropionic acid $(0.11 \mathrm{M})$ to $10 \mathrm{~mL}$ of water, formerly added with $1 \mathrm{~mL}$ of $\mathrm{HAuCl}_{4}(10 \mathrm{mM})$. The resultant mixture was stirred for $\sim 20$ min which led to the formation of a non-luminescent dispersion featuring the presence of ultrasmall nanoclusters under a transmission electron microscope.

\section{Instruments}

\section{Optical measurements}

UV-vis absorption spectra were acquired using an Agilent Cary 100 UV-vis spectrophotometer. Prompt fluorescence measurements were carried out with a HORIBA FluoroMax-4 spectrofluorometer. Horiba Jobin Yvon Fluoromax 4P was used for the acquisition of delayed fluorescence spectra. For measurement of delayed luminescence lifetime, the excitation and emission wavelengths were set at $300 \mathrm{~nm}$ and $494 \mathrm{~nm}$, respectively. The delayed luminescence spectrum was acquired over a time range of $400 \mu \mathrm{s}$ and the number of channels was fixed at 4000. An initial delay of $1 \mu \mathrm{s}$ was given to measure the long luminescence lifetime. The spectrum was acquired with a monochromator slit width of $6 \mathrm{~nm}$.

\section{Transmission electron microscopy measurements}

TEM measurements were performed using a JEOL JEM $2100 \mathrm{~F}$ at a maximum accelerating voltage of $200 \mathrm{kV}$.

\section{Fourier transformed infra-red spectral measurements}

FTIR measurements were performed using a PerkinElmer Spectrum Two - FTIR spectrometer.

\section{Zinc ion mediated aggregation of gold nanoclusters}

The as-synthesized clusters (as per the aforementioned protocol) were added to $\sim 200 \mathrm{mg}$ of zinc acetate dihydrate. This led to the appearance of a yellowish green color of the resultant dispersion which featured bright luminescence upon excitation at $300 \mathrm{~nm}$.

\section{Purification of $\mathrm{Zn}$ Au NCs}

The as-synthesized dispersion of $\mathrm{Zn}$ Au NCs was centrifuged at $10000 \mathrm{rpm}$ for 10 minutes. The supernatant was discarded and the as-obtained pellet was redispersed in water for further experiments. 


\section{Delayed fluorescence-based recognition of pyrophosphate}

$2 \mathrm{~mL}$ of the as-prepared $\mathrm{Zn} \mathrm{Au} \mathrm{NCs} \mathrm{was} \mathrm{taken} \mathrm{in} \mathrm{a} \mathrm{fluorescence}$ cuvette. To this dispersion, sequential volumes of sodium pyrophosphate solution (with concentrations as mentioned in the figure legends) were added and the corresponding delayed luminescence spectra were recorded.

\section{Control experiments}

The effect of interfering anions on the delayed luminescence of $\mathrm{Zn} \mathrm{Au} \mathrm{NCs} \mathrm{was} \mathrm{elucidated} \mathrm{in} \mathrm{a} \mathrm{manner} \mathrm{akin} \mathrm{to} \mathrm{pyrophosphate}$ recognition - keeping the concentration of $\mathrm{Zn} \mathrm{Au} \mathrm{NCs} \mathrm{and} \mathrm{all}$ other experimental conditions the same as that of pyrophosphate recognition.

\section{Sample preparation for TEM measurements}

In order to prepare samples for TEM measurements, the asprepared dispersion of $\mathrm{Zn} \mathrm{Au} \mathrm{NCs} \mathrm{and} \mathrm{that} \mathrm{following} \mathrm{the}$ addition of pyrophosphate ions were diluted 8 times. $7 \mu \mathrm{L}$ of the resultant dispersion was drop-cast onto a carbon coated copper grid and was dried overnight.

\section{Instrumental details for the measurement of delayed luminescence spectra}

For measurement of the delayed luminescence lifetime, the excitation and emission wavelengths were set at $300 \mathrm{~nm}$ and $494 \mathrm{~nm}$, respectively. The delayed luminescence spectrum was acquired over a time range of $400 \mu$ s and the number of channels was fixed at 4000. An initial delay of $1 \mu$ s was given to measure the long luminescence lifetime. The spectrum was acquired with a monochromator slit width of $6 \mathrm{~nm}$.

\section{Size distribution of nanoclusters}

In order to calculate the size of the nanoclusters, the sizes of $\sim 40$ such nanoclusters, from various TEM images, were measured using Gatan microscopy suite software. The as-obtained sizes of the particles were then plotted against the number of particles belonging to the specific size range using origin software.

\section{Conclusions}

In a nutshell, we have demonstrated the use of zinc induced assembly of gold nanoclusters as a delayed luminescence probe of pyrophosphate ions. Pyrophosphate anions were found to quench the delayed luminescence of $\mathrm{Zn}$ Au NCs. This signified that the quenching process arose due to a ground state phenomenon, possibly due to specific chemical interaction of the pyrophosphate ion with the zinc ions of $\mathrm{Zn} \mathrm{Au} \mathrm{NCs,} \mathrm{thereby}$ causing the disassembly of the nanoprobe followed by luminescence quenching.

\section{Conflicts of interest}

There are no conflicts to declare.

\section{Acknowledgements}

We would like to thank the Department of Electronics and Information Technology, Government of India, for financial support [No. 5(9)/2012-NANO (Vol. II)]. The Central Instruments Facility of Indian Institute of Technology Guwahati is acknowledged for providing instrumental facilities.

\section{Notes and references}

1 M. Dhiman, A. Maity, A. Das, R. Belgamwar, B. Chalke, Y. Lee, K. Sim, J.-M. Nam and V. Polshettiwar, Chem. Sci., 2019, 10, 6594-6603.

2 S. Izumi, H. F. Higginbotham, A. Nyga, P. Stachelek, N. Tohnai, P. de Silva, P. Data, Y. Takeda and S. Minakata, J. Am. Chem. Soc., 2020, 142, 1482-1491.

3 P. Rajamalli, N. Senthilkumar, P. Gandeepan, P.-Y. Huang, M.-J. Huang, C.-Z. Ren-Wu, C.-Y. Yang, M.-J. Chiu, L.-K. Chu, H.-W. Lin and C.-H. Cheng, J. Am. Chem. Soc., 2016, 138, 628-634.

4 Q. Ai, J. Chai, W. Lou, T. Liu, D. Wang, C. Deng, C. Wang, G. Li, X. Liu, Z. Liu and Q. Zhang, ACS Appl. Mater. Interfaces, 2020, 12, 6127-6136.

5 X. Liao, X. Yang, R. Zhang, J. Cheng, J. Li, S. Chen, J. Zhu and L. Li, J. Mater. Chem. C, 2017, 5, 10001-10006.

6 H. Yersin, L. Mataranga-Popa, R. Czerwieniec and Y. Dovbii, Chem. Mater., 2019, 31(16), 6110-6116.

7 K. Matsuoka, K. Albrecht, A. Nakayama, K. Yamamoto and K. Fujita, ACS Appl. Mater. Interfaces, 2018, 10, 33343-33352.

8 T. J. Penfold, F. B. Dias and A. P. Monkman, Chem. Commun., 2018, 54, 3926-3935.

9 P. Rajamalli, N. Senthilkumar, P.-Y. Huang, C.-C. Ren-Wu, H.-W. Lin and C.-H. Cheng, J. Am. Chem. Soc., 2017, 139, 10948-10951.

10 X.-K. Chen, S.-F. Zhang, J.-X. Fan and A.-M. Ren, J. Phys. Chem. C, 2015, 119, 9728-9733.

11 Y. Olivier, J.-C. Sancho-Garcia, L. Muccioli, G. D’Avino and D. Beljonne, J. Phys. Chem. Lett., 2018, 9, 6149-6163.

12 T. Li, D. Yang, L. Zhai, S. Wang, B. Zhao, N. Fu, L. Wang, Y. Tao and W. Huang, Adv. Sci., 2017, 4, 1600166.

13 S. Gan, J. Zhou, T. A. Smith, H. Su, W. Luo, Y. Hong, Z. Zhao and B. Zhong Tang, Mater. Chem. Front., 2017, 1, 2554-2558.

14 W. Hu, L. Guo, L. Bai, X. Miao, Y. Ni, Q. Wang, H. Zhao, M. Xie, L. Li, X. Lu, W. Huang and Q. Fan, Adv. Healthcare Mater., 2018, 7, 1870062.

15 X. Xiong, F. Song, J. Wang, Y. Zhang, Y. Xue, L. Sun, N. Jiang, P. Gao, L. Tian and X. Peng, J. Am. Chem. Soc., 2014, 136, 9590-9597.

16 Z. Zhu, D. Tian, P. Gao, K. Wang, Y. Li, X. Shu, J. Zhu and Q. Zhao, J. Am. Chem. Soc., 2018, 140, 17484-17491.

17 Q. Zhang, S. Xu, M. Li, Y. Wang, N. Zhang, Y. Guan, M. Chen, C.-F. Chen and H.-Y. Hu, Chem. Commun., 2019, 55, 5639-5642. 
18 X. Luo, J. Meng, B. Li, A. Peng and Z. Tian, New J. Chem., 2019, 43, 10735-10743.

19 P. Zhang, J. Zeng, J. Guo, S. Zhen, B. Xiao, Z. Wang, Z. Zhao and B. Z. Tang, Front. Chem., 2019, 7, 199.

20 J. Zeng, J. Guo, H. Liu, J. W. Y. Lam, Z. Zhao, S. Chen and B. Z. Tang, Chem. - Asian J., 2019, 14, 828.

21 F. Ni, Z. Zhu, X. Tong, M. Xie, Q. Zhao, C. Zhong, Y. Zou and C. Yang, Chem. Sci., 2018, 9, 6150-6155.

22 Y. H. Lee, S. Jana, H. Lee, S. U. Lee and M. H. Lee, Chem. Commun., 2018, 54, 12069-12072.

23 X. Li, G. Baryshnikov, C. Deng, X. Bao, B. Wu, Y. Zhou, H. Ågren and L. Zhu, Nat. Commun., 2019, 10, 731.

24 C. I. C. Crucho, J. Avó, A. M. Diniz, S. N. Pinto, J. Barbosa, P. O. Smith, M. N. Berberan-Santos, L.-O. Palsson and F. B. Dias, Front. Chem., 2020, 08, 202.

25 C. M. Tonge, N. R. Paisley, A. M. Polgar, K. Lix, W. R. Algar and Z. M. Hudson, ACS Appl. Mater. Interfaces, 2020, 12(5), 6525-6535.

26 C. Gayen, S. Basu, U. Goswami and A. Paul, Langmuir, 2019, 35(27), 9037-9043.
27 B. Li, X. Wang, X. Shen, W. Zhu, L. Xu and X. Zhou, J. Colloid Interface Sci., 2016, 467, 90-96.

28 S. Basu, A. Paul and A. Chattopadhyay, Chem. - Eur. J., 2017, 23, 9137.

29 J.-G. You, C.-Y. Lu, A. S. K. Kumar and W.-L. Tseng, Nanoscale, 2018, 10, 17691-17698.

30 F. Huo, Y. Liu, Y. Tang, Y. Cao, C. Tan, F. Yang and X. Yang, Colloids Surf., B, 2019, 183, 110335.

31 S. Basu, A. Paul and A. Chattopadhyay, J. Mater. Chem. A, 2016, 4, 1218-1223.

32 S. Basu, S. Bhandari, U. N. Pan, A. Paul and A. Chattopadhyay, J. Mater. Chem. C, 2018, 6, 8205-8211.

33 M. Paul, S. Basu and A. Chattopadhyay, Langmuir, 2020, 36(3), 754-759.

34 S. Basu, A. Hajra, C. Gayen and A. Paul, ChemPhysChem., 2020, 21, 809.

35 K. A. Jolliffe, Acc. Chem. Res., 2017, 50(9), 2254-2263.

36 Z. Cai, H. Chen, J. Guo, Z. Zhao and B. Z. Tang, Front. Chem., 2020, 8, 193. 